\title{
ECG Beat based Cardiac Disease Progression Monitoring for Wearables
}

\author{
P. Krishnan, Member, IEEE, V. Rajagopalan, and B.I. Morshed, Sr., Member, IEEE
}

\begin{abstract}
Real-time monitoring of cardiac disease progression using consumer electronics can be of immense benefit and realizable with the recent progress of wearables. This paper proposes a fast-acting personalized smart health wearable solution for cardiac disease monitoring using Electrocardiography (ECG). We also present a roadmap for a low-cost and low-power edge implementation of a smart health system. Premature Ventricular Contractions (PVC) are abnormal heartbeats and are prevalent in heart failure and other cardiac diseases. Our algorithm classifies ECG beats into Normal and PVC beat, and the severity of the PVC beat is grouped into borderline, mild, moderate, marked, severe, and extreme classes. Cardiac disease severity was validated using the Congestive Heart Failure (CHF) dataset consisting of data from 13 subjects (containing 1,208,778 ECG beats). ECG beat classification performance results from Arrhythmia and Normal Sinus Rhythm datasets consisting of 46 subjects (containing 1,697,851 ECG beats) had a combined accuracy, sensitivity, and specificity of $96.36 \%, 88.74 \%$, and $98.18 \%$, respectively. The algorithm was developed using model-based design and fuzzy logic. It is computationally efficient and easily portable to the smart-health device. The severity metric allows the consumer to easily track their cardiac health and helps in early detection and progression monitoring of cardiac diseases.
\end{abstract}

Index Terms - Cardiac Disease, electrocardiography (ECG), fuzzy logic, model-based design, smart health, wearables.

\section{INTRODUCTION}

$\mathrm{C}^{2}$ ardiac disease has high morbidity and mortality rate associated with factors such as population aging and delayed detection [1]. Due to the advent of newer consumer electronics like smartphones, wearables, smartwatches, and Internet-of-things (IoT), tracking of cardiac health has become a possibility. Cardiac arrhythmia-related ECG beats can be classified and monitored using a single electrode ECG wearable for data acquisition and a smartphone application for tracking cardiac health [2].

This work has been submitted to the IEEE for possible publication. Copyright may be transferred without notice, after which this version may no longer be accessible.

Manuscript received on September 18, 2020; revised November 24, 2020

P. Krishnan is an independent researcher in Electrical Engineering, Memphis, USA. (email: preethikrishnan27@gmail.com).

V. Rajagopalan is with the Department of Basic Sciences, New York Institute of Technology College of Medicine at Arkansas State University, Jonesboro, AR 72467 USA. (e-mail: vrajagop@ nyit.edu).

B. I. Morshed is with the Department of Computer Science, Texas Tech University, Lubbock, TX 79409 USA. (e-mail: bmorshed@ttu.edu)
Real-time data collected through remote monitoring can help in alerting clinicians and hospitals of any cardiac-related emergencies [3]. Smart health devices help in remote monitoring of ECG by using low-cost wireless data transmission systems such as Zigbee and Bluetooth. Long term ECG monitoring is possible using noncontact wireless wearables where dry capacitive coupled flexible electrodes are used to record data using smartphone [4].

One of the most common complications of cardiac disease is heart failure (HF). PVCs can represent a modifiable risk factor for Congestive Heart Failure (CHF) and death [5]. PVCs also give rise to ventricular arrhythmias such as ventricular fibrillation and ventricular tachycardia which are lifethreatening and can cause a sudden loss of consciousness and sudden cardiac death [6]. An increase in the number of PVC beats correlates with an increase in severity for HF which can lead to hospitalization and a higher mortality rate [7].

Clinicians divide HF into four classes using the New York Heart Association (NYHA) classification to identify gross changes in cardiac severity using routine daily activities. The A-D classifications were introduced to make the functional classification objective using ECG, X-rays, and radiological images. The subjective functional classification is performed using a questionnaire based on physical limitations. Class I patients have no limitations in physical activity. Class II, III, and IV indicate a progressive worsening of physical mobility where Class IV patients are always in discomfort during daily physical activities [8]. The NYHA classification has many drawbacks as explained below. The NYHA classification is not considered strictly as a functional assessment, as it is about the comparison of symptoms during the prognosis and the time that the same person was healthy [9]. The same subject reviewed by different physicians can be assigned different NYHA classes as there is no consensus on HF severity [10]. The perceived symptoms reported by the patient may be dependent on their psychological condition e.g. depression. Patients who generally lack clinical know-how may disregard or forget some symptoms that they experience. Studies have shown that women are more interactive during the questioning of their symptoms which puts the male subjects, who typically are at an increased risk for cardiovascular disease, at an even higher risk [11]. Clinical trials conducted by the National Institute of Health (NIH) have shown that NYHA classification is an unreliable metric for HF severity [9].

Wearable systems for detecting heart failure consist of three major blocks: sensor data acquisition from the wearable device, ECG beat classification, and an output block. HF is detected using heart rate variability with the help of sensors such as ECG, photoplethysmography (PPG), and accelerometer. HF 
subjects have less heart rate variability and the PPG sensor reflects oxygen saturation [12]. Heart rate estimation and accuracy of PPG and accelerometer can be improved using Adaptive Neural Network filtering [13]. ECG related heart rate and bioimpedance sensors to measure the pulmonary fluid have also been used to monitor HF [14]. Sensors that monitor potassium blood content, activity-related energy consumption, skin temperature, and sweating can alert the consumer during HF [15]. ECG beat classification can be performed using various techniques such as fuzzy logic [16], neuro-fuzzy [17], adaptive fuzzy neuro systems using Lyapunov exponents [18], and extreme learning machine algorithms [19]. The output block consists of plots or medical questionaries' regarding heart attack symptoms [20].

We propose a cardiac disease monitoring algorithm using single-lead ECG wearable which was developed using modelbased design and fuzzy logic. ECG signal is classified into a Normal beat and PVC beat and the algorithm further classifies PVC beat into a severity class. To our knowledge, there are no other consumer wearables that provide a personalized health solution by monitoring cardiac disease by using PVC beat severity. Historically, fuzzy logic has been widely used and trusted in intensive care, neurology, neurosurgery, cardiology, nephrology, hepatology, and pulmonology [21]. Fuzzy logic has been used for the development of wearable systems [22]. Contemporary methods like deep learning which is a black box methodology are resource intensive. The combination of model-based design and fuzzy logic allows our code to be lightweight. The model-based design allows for easy portability of embedded code to a smart health device. e.g. microcontroller or smartphone application [23].

Severity ranking for atrial fibrillation using a random forest classifier has been successful as shown in our previous works [24]. In our proof of concept study, we have shown that severity classification using the fuzzy output (Rule Firing Strength RFS) can be used to classify PVC beats. The study used a total of 3880 ECG beats [25]. In this work, we have optimized our fuzzy algorithm and the ECG beat classification has been verified using ECG data from Arrhythmia and Normal Sinus Rhythm dataset. PVC severity classification has been verified using ECG data from Congestive Heart Failure (CHF) subjects. The CHF dataset chosen for this study has been classified by the NYHA metric thereby allowing a comparison of NYHA to our severity metric. A total of 2.9 million ECG beats from 59 patients were used to evaluate our algorithm.

\section{METHODOLOGY}

\section{A. Cardiac Disease Monitoring System Roadmap}

Our proposed system will consist of an ECG wearable with edge-computation and a smartphone application for userinteraction. The code is computationally simple, and the entire software code can be ported into the wearable device. The software application will consist of two blocks: ECG beat classification and PVC beat severity classification. The algorithm uses model-based design and an efficient embedded code can be generated automatically. This reduces the development time required to create the software. Using Bluetooth or Zigbee protocol, the severity output will be sent to a smartphone application. The system can detect and classify
PVC beat into a severity class such as borderline, mild, moderate, marked, severe, and extreme classes. Two of the classes (severe and extremely severe) require immediate attention from the consumer and their clinician. During an HF episode, all relevant severity data can be remotely transferred to a hospital or clinic thus making the system compatible with personalized medicine and telehealth.

\section{B. ECG Features}

The electrical activity of the heart is represented by the ECG signal which consists of three major components: P-wave, the QRS complex, and the T wave as shown in Fig. 1.

The first positive deflection on the ECG waveform is defined

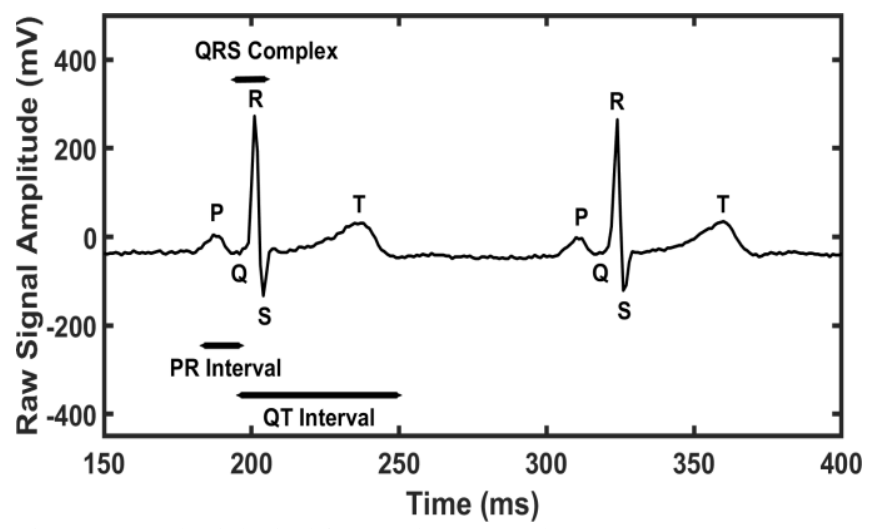

Fig. 1. ECG physiological features in a Normal Beat.

as the $\mathrm{P}$ wave which represents the depolarization of atria (upper chambers of the heart). PR interval represents the time between the onset of depolarization of atria and the onset of depolarization of ventricles (lower chambers of the heart), in an ECG strip. PR interval corresponds to the time from the onset of the $\mathrm{P}$ wave to the beginning of the $\mathrm{QRS}$ complex. While the QRS complex represents ventricular depolarization, T-wave represents ventricular repolarization. The QRS complex duration is defined as the time interval between the start and end of the QRS complex. The QT interval is measured from the beginning of QRS to the end of the T wave and represents the time for both ventricular depolarization and repolarization.

ECG beats can have abnormalities at multiple levels, i.e., atria, atrioventricular, nodal, ventricular. Healthy individuals predominantly have normal beats $(\mathrm{N})$ but may also have PVCs. However, the larger percentage of PVCs can be seen in conditions such as heart failure, ventricular tachycardia, and ventricular fibrillation, and are also clinically considered to be early biomarkers of potential cardiac diseases [26]. A Normal beat includes the presence of P-wave, QRS complex, PR interval, and QT interval within normal ranges. The defining features of the PVC beat include the absence of $\mathrm{P}$ wave, wide QRS complex, and non-measurable PR interval [27]-[29]. The details are tabulated in the subsequent sections in Table I, II, III, and IV.

\section{Cardiac Disease Monitoring Algorithm}

Fuzzy logic is a linguistic rule-based system to classify ECG beats into Normal or PVC beats. The algorithm was implemented using a model-based Fuzzy Inference System. 
The fuzzy inference system consists of a fuzzy logic designer, membership function editor, and rule editor. A Sugeno type fuzzy model was created using the fuzzy logic designer. Sugeno type models are computationally efficient and guarantees output space continuity. They also work well with optimization, adaptive techniques, and mathematical analysis. The proposed Cardiac Disease Monitoring Algorithm is shown in Fig. 2. The algorithm consists of four stages: Wavelet delineation, ECG physiological features, ECG Beat Fuzzy Classifier, and Severity Index Classifier.

Stage 1 - ECG data: Single lead ECG data was considered for ECG beat classification. Data was used from either Modified Lead II (MLII) or ECG1. ECG peaks (P, Q, R, S, T) were generated using the wavelet-based ECG delineation algorithm [30]. Raw ECG data is used as an input to the wavelet algorithm. Wavelet-based ECG delineation is performed using ECG-Kit software available on the Physionet [31].

Stage 2 - ECG physiological features: Noise removal is performed before the creation of the ECG physiological features. Values that are out of the normal range of ECG peak values $(\mathrm{P}, \mathrm{Q}, \mathrm{R}, \mathrm{S}, \mathrm{T})$ are removed. The ECG peak values are used to create ECG physiological features that are used as inputs to the Fuzzy Model ECG Beat Classifier. The inputs were chosen based on the usage by clinicians to diagnose ventricular arrhythmias. The input ECG features consist of QT interval, $\mathrm{P}$ wave, QRS complex interval, and PR interval.

Stage 3 - Fuzzy Model ECG Beat Classifier: The classifier was designed using the fuzzy inference process which consists of five parts: input fuzzification, a fuzzy operation using AND logic, fuzzy implication, aggregation, and defuzzification. Two separate models were utilized to classify the ECG beats into Normal or PVC beats. Each ECG feature input is fuzzified using membership functions. The input ECG features are mapped to fuzzy sets using membership functions. The fuzzified input values for all features lie between 0 and 1 . The membership functions used for the classifiers are Gaussian or Triangular. QRS complex and the P-wave absence/presence membership functions are shown in Fig. 3. Details of the input membership functions are provided in Table I and Table II. The membership functions were calibrated using medical literature
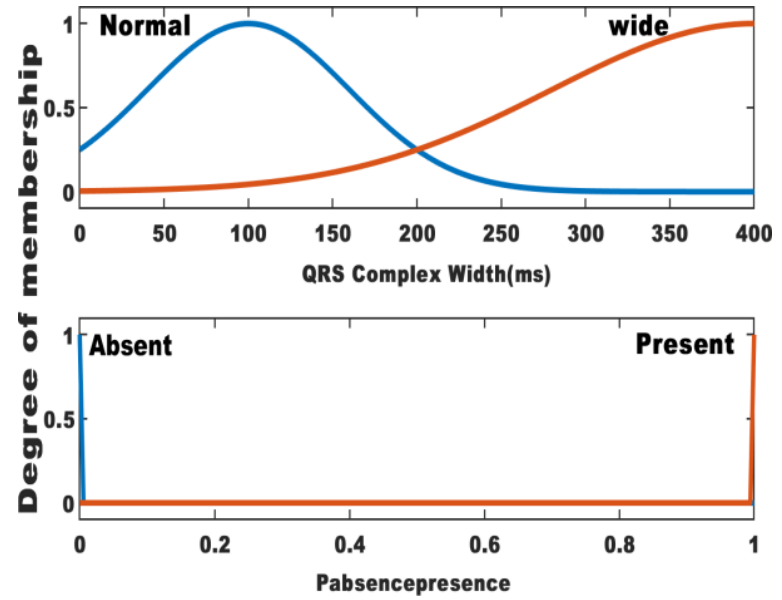

Fig. 3. Input membership function for QRS Complex and $\mathrm{P}$ wave absence / presence.

TABLE I

FUZZY INPUT MEMBERSHIP FUNCTION TYPE

\begin{tabular}{ccccc}
\hline \hline $\begin{array}{c}\text { Heartbeat } \\
\text { Type }\end{array}$ & $\begin{array}{c}\text { QT } \\
\text { Interval }\end{array}$ & P-wave & $\begin{array}{c}\text { QRS } \\
\text { Complex }\end{array}$ & $\begin{array}{c}\text { PR } \\
\text { Interval }\end{array}$ \\
\hline Normal & Gaussian & Trimf & Gaussian & Gaussian \\
PVC & Gaussian & Trimf & Gaussian & Gaussian \\
\hline \hline
\end{tabular}

TABLE II

FUZZY INPUT MEMBERSHIP FUNCTION VALUES

\begin{tabular}{ccccc}
\hline \hline $\begin{array}{c}\text { Heartbeat } \\
\text { Type }\end{array}$ & $\begin{array}{c}\text { QT - } \\
\text { Interval } \\
(\mathrm{ms})\end{array}$ & P-wave & $\begin{array}{c}\text { QRS } \\
\text { Duration } \\
(\mathrm{ms})\end{array}$ & $\begin{array}{c}\text { Interval } \\
(\mathrm{ms})\end{array}$ \\
\hline Normal & {$\left[\begin{array}{llll}100 & 450\end{array}\right]$} & {$\left[\begin{array}{lll}1 & 1 & 1\end{array}\right]$} & {$\left[\begin{array}{lll}60 & 100\end{array}\right]$} & {$\left[\begin{array}{lll}225 & 900\end{array}\right]$} \\
PVC & {$\left[\begin{array}{llll}310 & 440\end{array}\right]$} & {$\left[\begin{array}{lll}0 & 0 & 0\end{array}\right]$} & {$\left[\begin{array}{lll}82.5 & 137.5\end{array}\right]$} & {$\left[\begin{array}{lll}0 & 0 & 0\end{array}\right]$} \\
\hline \hline
\end{tabular}

TABLE III

FUZZY RULES FOR INPUT MEMEBERSHIP FUNCTIONS

\begin{tabular}{ccccl}
\hline \hline $\begin{array}{c}\text { QT } \\
\text { Interval }\end{array}$ & P-wave & $\begin{array}{c}\text { QRS Complex } \\
\text { Interval }\end{array}$ & $\begin{array}{c}\text { PR } \\
\text { Interval }\end{array}$ & $\begin{array}{c}\text { Heartbeat } \\
\text { Output }\end{array}$ \\
\hline Normal & Present & Normal & Normal & Normal \\
Normal & Absent & Wide & Absent & PVC
\end{tabular}

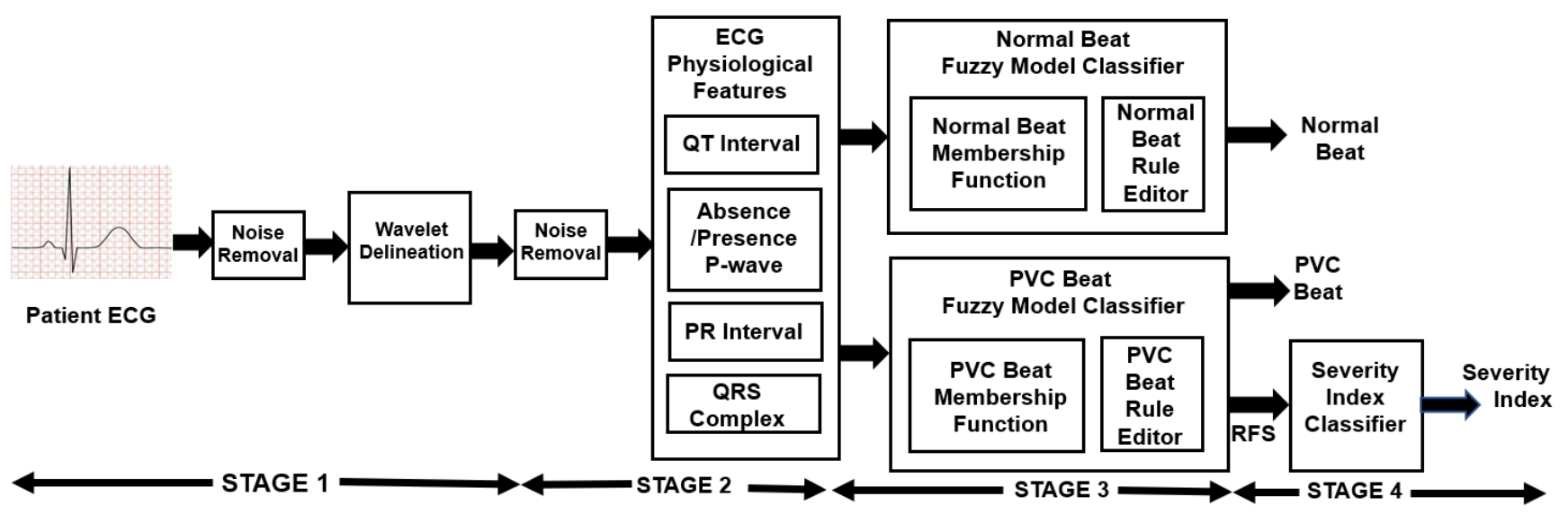

Fig. 2. Cardiac disease monitoring algorithm block diagram 
and discussions with a cardiovascular scientist. The linguistic rules for our fuzzy logic algorithm consist of IF-THEN rules derived from cardiac arrhythmia medical literature. The rules of the fuzzy system are defined in Table III. Rule weights are assigned to every ECG feature input before fuzzy implication and the values are between 0 and 1 . The output of each rule for the classifier is a single output fuzzy set. Defuzzification is performed using the sum aggregation method to obtain final fuzzy values which will classify an output to either Normal or PVC beat. As the Sugeno type of fuzzy model was chosen to design the algorithm, the product implication method was used. The output membership functions are defined in Table IV.

Stage 4 - Severity Index Classifier: Rule Firing Strength (RFS) is an output of the fuzzy classifier system which is computed by applying the fuzzy operator to the values of the fuzzified inputs. RFS is used as an input to the severity index classifier which classifies a range of RFS values into a severity class descriptor index as shown in Table $\mathrm{V}$.

\section{RESULTS}

ECG data from subjects and healthy individuals have been used as an input to the Fuzzy ECG Beat Classifier. The dataset from Physionet namely MIT (Massachusetts Institute of Technology) Arrhythmia and MIT Normal Sinus Rhythm databases from Beth Israel Hospital (BIH) were used to validate the ECG beat classification [31]. MIT-BIH Arrhythmia database contains 30-minute, $360 \mathrm{~Hz}$ sampling frequency ECG data obtained from cardiac patients. The database contains male and female patients with a large variety of Arrhythmias. For this study, 29 patients from a total of 47 were considered. Any patients predominantly with Bundle Branch beats, Fusion beats, Premature Atrial Contraction beats or $\mathrm{P}$ wave abnormalities were excluded as our focus is on classification and severity detection of PVC beats. As patients have been receiving treatment for their Arrhythmias, some patients do not exhibit PVCs or exhibit a varying number of PVC beats depending on cardiac disease severities, other comorbidities, and treatment compliance. MIT Arrhythmia database has been classified as a Physionet Class I database which indicates that annotations for ECG beats are available from two cardiologists as ground truth for comparing with the Fuzzy ECG Beat Classifier output. The Accuracy, Sensitivity, and Specificity for the 29 patients are shown in Table VI.
TABLE IV

FUZZY OUTPUT MEMBERSHIP FUNCTIONS

\begin{tabular}{|c|c|c|}
\hline Heartbeat Type & Type & Parameter \\
\hline Normal & Linear & {$\left[\begin{array}{llllll}1 & 1 & 1 & 1 & 1\end{array}\right]$} \\
\hline PVC & Linear & {$\left[\begin{array}{lllll}0.1 & 0.5 & 1 & 0.5 & 0.5\end{array}\right]$} \\
\hline \multicolumn{3}{|c|}{$\begin{array}{c}\text { TABLE V } \\
\text { PVC CLINICAL DESCRIPTORS } \\
\end{array}$} \\
\hline Clinical Descriptor & & RFS ranges \\
\hline Borderline (B) & & $1 \leq \mathrm{RFS}<10$ \\
\hline Mild (Mi) & & $10 \leq$ RFS $<15$ \\
\hline Moderate (Mo) & & $15 \leq \mathrm{RFS}<20$ \\
\hline Marked (Ma) & & $20 \leq \mathrm{RFS}<25$ \\
\hline Severe (S) & & $25 \leq \mathrm{RFS}<30$ \\
\hline Extreme (E) & & $\mathrm{RFS} \geq 30$ \\
\hline
\end{tabular}

The Fuzzy ECG beat classifier was also verified using MITBIH Normal Sinus Rhythm which contains 18 long-term ECG recordings from healthy subjects which include 5 men and 13 women. The records are 24 hours long and are sampled at 128 Hz. This database is classified as a Physionet Class III database i.e. there are no beat-by-beat reference annotations provided by cardiologists. Out of a total of 18 patients, 17 patients were considered for this study. Patient \# 16272 was excluded from

TABLE VIII

\begin{tabular}{ccc}
\multicolumn{3}{c}{ TABLE VIII } \\
\hline PERFORMANCE COMPARISON \\
\hline Classifier & $\begin{array}{c}\text { Sensitivity } \\
(\%)\end{array}$ & $\begin{array}{c}\text { Specificity } \\
(\%)\end{array}$ \\
\hline $\begin{array}{c}\text { Model based fuzzy } \\
\text { (this work) }\end{array}$ & 88.74 & 98.18 \\
Neuro fuzzy [17] & 97.12 & \\
$\begin{array}{c}\text { Fuzzy logic system } \\
\text { [16] }\end{array}$ & 81.34 & - \\
Deep learning [32] & 96.52 \\
Wavelet- SVM [33] & 99.09 & - \\
\hline \hline
\end{tabular}

TABLE VI

MIT-BIH ARRHYTHMIA DATABASE PERFORMANCE

\begin{tabular}{cccccccc}
\hline \hline Database & Patients & $\begin{array}{c}\text { ECG Duration } \\
\text { per Patient }(\mathrm{min})\end{array}$ & $\begin{array}{c}\text { Total } \\
\text { Beats }\end{array}$ & $\begin{array}{c}\text { Total Normal } \\
\text { Beats }\end{array}$ & $\begin{array}{c}\text { Total PVC } \\
\text { Beats }\end{array}$ & $\begin{array}{c}\text { Accuracy } \\
(\%)\end{array}$ & $\begin{array}{c}\text { Sensitivity } \\
(\%)\end{array}$ \\
\hline MIT Arrhythmia & 29 & 30 & 60653 & 56554 & 4099 & 96.87 \\
\hline \hline
\end{tabular}

TABLE VII

MIT-BIH NORMAL SINUS RHYTHM DATABASE PERFORMANCE

\begin{tabular}{cccccccc}
\hline \hline Database & Patients & $\begin{array}{c}\text { ECG Duration } \\
\text { per Patient (hours) }\end{array}$ & $\begin{array}{c}\text { Total } \\
\text { Beats }\end{array}$ & $\begin{array}{c}\text { Normal Beats } \\
\text { Detected }\end{array}$ & $\begin{array}{c}\text { Normal Beats } \\
\text { Detected }(\%)\end{array}$ & $\begin{array}{c}\text { Other } \\
\text { Beats }\end{array}$ & $\begin{array}{c}\text { Other } \\
\text { Beats }(\%)\end{array}$ \\
\hline MIT Normal Sinus Rhythm & 17 & 24 & $1,637,198$ & $1,569,020$ & 95.86 & 68435 & 4.14 \\
\hline \hline
\end{tabular}


our study as the ECG data had predominantly noisy artifacts. The performance of our classifier for detecting normal beats is given in Table VII. The model-based fuzzy classifier was compared to other ECG beat classifiers such as neuro-fuzzy [17], fuzzy logic system [16], deep learning [32], and waveletSupport Vector Machine (SVM) [33] in Table VIII.

BIDMC CHF database is a Class III database available on Physionet that contains long-term ECG from patients with severe CHF, NYHA class 3-4. The subjects include 11 men and 4 women. The subjects were receiving medical therapy for the treatment of CHF. The recordings are 20 hours long, sampled at $250 \mathrm{~Hz}$. CHF Patient \# 4 and CHF Patient \# 5 were excluded from our study as they did not exhibit any PVC beat. Every PVC detected in a CHF patient is classified into a severity class and the data from all patients have been tabulated in Table IX. To validate our severity classifier, we used clinical studies that showed that the elongation of the QRS interval leads to a higher mortality rate [7], [34], [35]. RFS range for every decade (1-10, 11-20, etc.) and the corresponding averaged values of the QRS complex were plotted for $13 \mathrm{CHF}$ patients as shown in Fig. The equation for RFS and corresponding QRS complex duration of all PVC beats are also specified in Fig. 4.

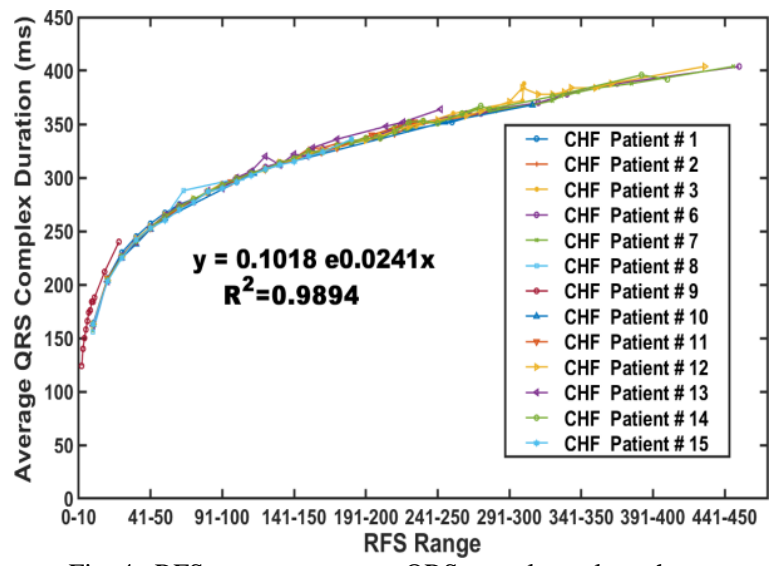

Fig. 4. RFS range vs average QRS complex values shows correlation

TABLE IX

CHF DATABASE PVC BEAT SEVERITY CLASSIFICATION

\begin{tabular}{|c|c|c|c|c|c|c|c|c|c|}
\hline Patient ID & $\begin{array}{l}\text { Total } \\
\text { Beats }\end{array}$ & $\begin{array}{c}\text { Normal } \\
\text { Beats }\end{array}$ & $\begin{array}{l}\text { Total } \\
\text { PVC } \\
\text { Beats }\end{array}$ & Borderline & Mild & Moderate & Marked & Severe & Extreme \\
\hline CHF Patient \# 1 & 72163 & 71113 & 1050 & 543 & 52 & 44 & 48 & 65 & 298 \\
\hline CHF Patient \# 2 & 112025 & 104096 & 7929 & 7553 & 65 & 32 & 31 & 21 & 227 \\
\hline CHF Patient \# 3 & 72958 & 59488 & 13470 & 8691 & 2628 & 808 & 385 & 192 & 766 \\
\hline CHF Patient \# 6 & 89651 & 88683 & 968 & 549 & 48 & 37 & 27 & 23 & 284 \\
\hline CHF Patient \# 7 & 88537 & 87402 & 1135 & 717 & 53 & 34 & 30 & 23 & 278 \\
\hline CHF Patient \# 8 & 86668 & 85946 & 722 & 640 & 30 & 13 & 10 & 9 & 20 \\
\hline CHF Patient \# 9 & 112000 & 111792 & 208 & 205 & 1 & 1 & 0 & 1 & 0 \\
\hline CHF Patient \# 10 & 143061 & 141068 & 1993 & 1887 & 46 & 21 & 9 & 6 & 24 \\
\hline CHF Patient \# 11 & 69177 & 66882 & 2295 & 661 & 554 & 754 & 182 & 37 & 107 \\
\hline CHF Patient \# 12 & 58321 & 41774 & 16547 & 1405 & 693 & 514 & 975 & 816 & 12144 \\
\hline CHF Patient \# 13 & 113219 & 112382 & 837 & 701 & 18 & 25 & 14 & 17 & 62 \\
\hline CHF Patient \# 14 & 82182 & 53377 & 28805 & 22204 & 4106 & 669 & 360 & 236 & 1230 \\
\hline CHF Patient \# 15 & 108816 & 105068 & 3748 & 3271 & 153 & 84 & 58 & 29 & 153 \\
\hline
\end{tabular}

\section{DISCUSSION}

Our methodology of cardiac monitoring uses a single ECG sensor. Additional sensors are not needed for our approach as HF is not detected using activity, temperature, or any other physiological signals. The consumer does not need to answer any questions for HF prediction. This makes our metric objective and user friendly as consumers may be in physical distress in cardiac emergencies. As the severity index classification is a critical heart monitoring system, the use of fuzzy logic, which is a white box methodology, will lead to improved understanding of the intermediate variables and algorithm outputs for both the clinical and engineering stakeholders thereby increasing trust and reliance on the automated outcome. Variability among different groups dependent on gender, age, etc. can be easily addressed by changing the fuzzy logic input membership functions. The fuzzy logic algorithm provides a computationally efficient design wherein the fuzzy rules can be derived directly from medical literature. The smaller set of input features used for our 
algorithm reduces the computational complexity. As a white box methodology, engineers and scientists during development will have access to inputs, outputs, and intermediate variables. Errors can be tracked easily through the system thereby increasing the reliability of our algorithm.

Hardware implementation of popular techniques like deep learning in wearables suffers from system overhead due to increased need for memory, computation, and energy [36]. Data can be transmitted to a wearable device in three ways: real-time transmission of all data, periodic transmission of data, or eventbased transmission. Energy consumption is directly related to data transmission used by the wearable [37]. As our proposed system uses only event-based data transmission, the amount of energy consumed by our system will be lower. The wearable relays the severity metric output to the smartphone making our product a low-power application. Memory consumption is also reduced as fuzzy logic is a lightweight implementation. Modelbased development can be used for rapid prototyping and can help in reducing firmware development time due to auto code generation capability. Software metrics like cyclomatic complexity can be easily computed using benchmarking tools. Overall memory reduction can also be a possible advantage as auto-generated code is highly optimized and avoids pitfalls usually created by human programming errors.

Our novel method of using model-based fuzzy logic has shown specificity of $98.18 \%$, sensitivity of $88.74 \%$, and accuracy of $96.87 \%$. The sensitivity value did not reach as high as the specificity partly due to 2 patients. Patient \# 234 had only 3 PVC beats totally and our algorithm detected two of them successfully. Patient \# 201 exhibited complex ECG abnormalities including atrial fibrillation, nodal rhythm, and non-conducted P-waves which may have affected the sensitivity. Nonetheless, our results show a significantly higher sensitivity level compared to other PVC detection studies using a fuzzy model [16] that showed a sensitivity of $81.34 \%$ and a positive predictive value of $80.64 \%$. The sensitivity of the fuzzy algorithm can be further improved by incorporating principal component analysis (PCA) and Support Vector Machine to our ECG beat classification wavelet transform. A wavelet algorithm is an efficient tool for ECG beat classification. However, some of the wavelet coefficients can contain redundant information. Using PCA and SVM can help with the dimensionality issue and improve overall sensitivity [33]. Our algorithm was also able to detect PVCs in healthy subjects within the acceptable range of $1 \%-4 \%$ as observed in published clinical studies [38].

Our HF classification system goes beyond the four NYHA classes and can further dissect and offer a subtler distinction of patients into different severity groups. PVC severity classification was validated by using clinical QRS complex data as widely used in mortality studies. The QRS complex duration and RFS data from 13 CHF patients showed identical trends. Patients in NYHA Class III have a marked limitation of physical activity and comfortable at rest. NYHA Class IV indicates the inability to carry on any physical activity without discomfort. The entire CHF database was classified as NYHA Class III / Class IV. Our classification shows that all 12 CHF patients had extreme PVC beats (RFS $\geq 30$ ). Patients in a clinical study may respond differently even when the same drug is administered. CHF Patient \# 4 and CHF Patient \# 5 did not have any PVC beats. CHF Patient \# 9 has predominately borderline PVC beats. The treatment may have been more effective for certain patients as seen in CHF Patient \# 4, CHF Patient \# 5, and CHF Patient \# 9. On the contrary CHF Patient \# 12 has many extreme PVC beats. However, the NYHA classification system has been shown to poorly discriminate against HF patients across the spectrum of functional impairment. Also, the classification is based on judgment as there is no clear consensus on the usage of the terms minimal, moderately severe, and severe disease [9]. All the above-given limitations of NYHA classification can be overcome by using a reliable metric that does not have any subjective discrepancy. As a health monitoring system, the grouping of PVCs into distinct classes like borderline, mild, moderate, marked severe, and extreme will help consumers track cardiac health and receive urgent care during cardiac medical emergencies.

\section{CONCLUSION}

In this work, we have shown a smart health progression monitoring system for cardiac disease. The advantage of our approach is that the system uses a single ECG sensor thereby lowering the cost of the overall electronics system. The use of fewer clinical rules makes it a superior choice compared to other complex statistical approaches and helps in the reduction of power consumption in the wearable device. Our method of using model-based design will help in the rapid prototyping of Smart Health wearables. The proposed automated algorithm is not a subjective classification system as with NYHA and is reliable and reproducible. The algorithm can be easily implemented in a smart health wearable device for real-time operation with streaming data. The proposed algorithm is computationally efficient, user-friendly, and easily tunable thus suitable for remote health, personalized medicine, and telemedicine. Furthermore, our novel approach can be extended to several other life-threatening and benign cardiac arrhythmias.

\section{REFERENCES}

[1] S. S. Virani et al., "Heart Disease and Stroke Statistics-2020 Update: A Report from the American Heart Association," Circulation, vol. 141, no. 9, pp. e139-e596, 032020.

[2] S. Y. Lee, P. W. Huang, M. C. Liang, J. H. Hong, and J. Y. Chen, "Development of an Arrhythmia Monitoring System and Human Study," IEEE Trans. Consumer Electron., vol. 64, no. 4, pp. 442-451, Nov. 2018.

[3] N. Dey, A. S. Ashour, F. Shi, S. J. Fong, and R. S. Sherratt, "Developing residential wireless sensor networks for ECG healthcare monitoring," IEEE Trans. Consumer Electron., vol. 63, no. 4, pp. 442-449, Nov. 2017.

[4] S. Majumder, L. Chen, O. Marinov, C.-H. Chen, T. Mondal, and M. J. Deen, "Noncontact Wearable Wireless ECG Systems for Long-Term Monitoring,” IEEE Rev. Biomed. Eng., vol. 11, pp. 306-321, 2018.

[5] J. W. Dukes et al., "Ventricular Ectopy as a Predictor of Heart Failure and Death," J Am Coll Cardiol, vol. 66, no. 2, pp. 101-109, Jul. 2015.

[6] B. Lown and M. Wolf, "Approaches to sudden death from coronary heart disease," Circulation, vol. 44, no. 1, pp. 130-142, Jul. 1971.

[7] C. Y. Lin et al., "An observational study on the effect of premature ventricular complex burden on long-term outcome," Medicine (Baltimore), vol. 96, no. 1, p. e5476, Jan. 2017.

[8] C. Miller-Davis, S. Marden, and N. K. Leidy, "The New York Heart Association Classes and functional status: what are we really measuring?," Heart Lung, vol. 35, no. 4, pp. 217-224, Aug. 2006.

[9] J. A. Bennett, B. Riegel, V. Bittner, and J. Nichols, "Validity and reliability of the NYHA classes for measuring research outcomes in 
patients with cardiac disease," Heart Lung, vol. 31, no. 4, pp. 262-270, Aug. 2002.

[10] C. Raphael et al., "Limitations of the New York Heart Association functional classification system and self-reported walking distances in chronic heart failure," Heart, vol. 93, no. 4, pp. 476-482, Apr. 2007.

[11] K. M. Goode, S. Nabb, J. G. F. Cleland, and A. L. Clark, "A comparison of patient and physician-rated New York Heart Association class in a community-based heart failure clinic," J Card Fail, vol. 14, no. 5, pp. 379-387, Jun. 2008.

[12] A. J. Shah, N. Isakadze, O. Levantsevych, A. Vest, G. Clifford, and S. Nemati, "Detecting heart failure using wearables: a pilot study," Physiol Meas, vol. 41, no. 4, p. 044001, 042020.

[13] S. Puranik and A. W. Morales, "Heart Rate Estimation of PPG Signals with Simultaneous Accelerometry Using Adaptive Neural Network Filtering," IEEE Trans. Consumer Electron., vol. 66, no. 1, pp. 69-76, Feb. 2020.

[14] S. Lee et al., "Congestive heart failure patient monitoring using wearable Bio-impedance sensor technology," in 2015 37th Annual International Conference of the IEEE Engineering in Medicine and Biology Society (EMBC), Milan, Aug. 2015, pp. 438-441.

[15] H. Solar et al., "A Non Invasive, Wearable Sensor Platform for Multiparametric Remote Monitoring in CHF Patients," in Impact Analysis of Solutions for Chronic Disease Prevention and Management, vol. 7251, M. Donnelly, C. Paggetti, C. Nugent, and M. Mokhtari, Eds. Berlin, Heidelberg: Springer Berlin Heidelberg, 2012, pp. 140-147.

[16] O. Wieben, W. J. Tompkins, and V. X. Afonso, "Classification of PVCs with a fuzzy logic system," in Proceedings of the 19th Annual International Conference of the IEEE Engineering in Medicine and Biology Society. "Magnificent Milestones and Emerging Opportunities in Medical Engineering", Chicago, IL, USA, 1997, vol. 1, pp. 65-67.

[17] M. A. Chikh, M. Ammar, and R. Marouf, "A neuro-fuzzy identification of ECG beats," J Med Syst, vol. 36, no. 2, pp. 903-914, Apr. 2012.

[18] E. D. Ubeyli, "Adaptive neuro-fuzzy inference system for classification of ECG signals using Lyapunov exponents," Comput Methods Programs Biomed, vol. 93, no. 3, pp. 313-321, Mar. 2009.

[19] J. Kim, H. Shin, K. Shin, and M. Lee, "Robust algorithm for arrhythmia classification in ECG using extreme learning machine," BioMed Eng OnLine, vol. 8, no. 1, p. 31, 2009.

[20] P. Leijdekkers and V. Gay, "A Self-Test to Detect a Heart Attack Using a Mobile Phone and Wearable Sensors," in 2008 21st IEEE International Symposium on Computer-Based Medical Systems, Jyvaskyla, Finland, Jun. 2008, pp. 93-98.

[21] S. Thukral and V. Rana, "Versatility of fuzzy logic in chronic diseases: A review," Medical Hypotheses, vol. 122, pp. 150-156, Jan. 2019.

[22] C. C. Oliveira and J. Machado da Silva, "A fuzzy logic approach for highly dependable medical wearable systems," in 2015 IEEE 20th International Mixed-Signals Testing Workshop (IMSTW), Paris, France, Jun. 2015, pp. 1-5.

[23] A. Ruiz-Zafra, M. Noguera, K. Benghazi, and S. F. Ochoa, "A ModelDriven Approach for Wearable Systems Developments," International Journal of Distributed Sensor Networks, vol. 2015, pp. 1-12, 2015.

[24] H. Gollakota and B. I. Morshed, "A Novel Severity Ranking Approach for Continuous Monitoring of Heart Disease Progression Using Beatwise Classification of ECG," in 2019 IEEE International Conference on Electro Information Technology (EIT), Brookings, SD, USA, May 2019, pp. 261-265.

[25] P. Krishnan, V. Rajagopalan, and B. I. Morshed, "ANovel Severity Index of Heart Disease from Beat-wise Analysis of ECG Using Fuzzy Logic for Smart-Health," in 2020 IEEE International Conference on Consumer Electronics (ICCE), Las Vegas, NV, USA, Jan. 2020, pp. 1-5.

[26] S. H. Sheldon, J. J. Gard, and S. J. Asirvatham, "Premature Ventricular Contractions and Non-sustained Ventricular Tachycardia: Association with Sudden Cardiac Death, Risk Stratification, and Management Strategies," Indian Pacing Electrophysiol J, vol. 10, no. 8, pp. 357-371, Aug. 2010.

[27] J. Huff, ECG Workout, 3rd Edition, Lippincott, 1997.

[28] G. M. Marcus, "Evaluation and Management of Premature Ventricular Complexes," Circulation, vol. 141, no. 17, pp. 1404-1418, Apr. 2020.

[29] S. M. Al-Khatib et al., "2017 AHA/ACC/HRS Guideline for Management of Patients with Ventricular Arrhythmias and the Prevention of Sudden Cardiac Death: A Report of the American College of Cardiology/American Heart Association Task Force on Clinical Practice Guidelines and the Heart Rhythm Society," J Am Coll Cardiol, vol. 72, no. 14, pp. e91-e220, 022018.
[30] J. P. Martinez, R. Almeida, S. Olmos, A. P. Rocha, and P. Laguna, "A Wavelet-Based ECG Delineator: Evaluation on Standard Databases," IEEE Trans. Biomed. Eng., vol. 51, no. 4, pp. 570-581, Apr. 2004.

[31] A. L. Goldberger et al., "PhysioBank, PhysioToolkit, and PhysioNet: components of a new research resource for complex physiologic signals," Circulation, vol. 101, no. 23, pp. E215-220, Jun. 2000

[32] W. Zhao et al., "Deep Learning Based Patient-Specific Classification of Arrhythmia on ECG signal," in 201941 st Annual International Conference of the IEEE Engineering in Medicine and Biology Society (EMBC), Berlin, Germany, Jul. 2019, pp. 1500-1503.

[33] Q. Qin, J. Li, L. Zhang, Y. Yue, and C. Liu, "Combining Lowdimensional Wavelet Features and Support Vector Machine for Arrhythmia Beat Classification,” Sci Rep, vol. 7, no. 1, p. 6067, 202017.

[34] S. L. Hummel, S. Skorcz, and T. M. Koelling, "Prolonged electrocardiogram QRS duration independently predicts long-term mortality in patients hospitalized for heart failure with preserved systolic function," J Card Fail, vol. 15, no. 7, pp. 553-560, Sep. 2009.

[35] M. Guglin and A. B. Curtis, "Cardiac resynchronization therapy: 150 is not a magic number!," Circ Arrhythm Electrophysiol, vol. 6, no. 2, pp. 429-435, Apr. 2013.

[36] N. D. Lane, S. Bhattacharya, P. Georgiev, C. Forlivesi, and F. Kawsar, "An Early Resource Characterization of Deep Learning on Wearables, Smartphones and Internet-of-Things Devices," in Proceedings of the 2015 International Workshop on Internet of Things towards Applications - IoT-App '15, Seoul, South Korea, 2015, pp. 7-12.

[37] J. Y. Kim and C. H. Chu, "Analysis of energy consumption for wearable ECG devices," in IEEE SENSORS 2014 Proceedings, Valencia, Spain, Nov. 2014, pp. 962-965.

[38] J. B. Kostis et al., "Premature ventricular complexes in the absence of identifiable heart disease," Circulation, vol. 63, no. 6, pp. 1351-1356, Jun. 1981

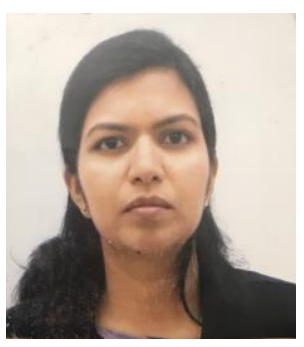

Preethi Krishnan completed her bachelor's degree in Electrical and Electronics Engineering from Jawaharlal Nehru Technological University (India) in 2005 and master's degree in Electrical and Engineering from Texas A and M University, Kingsville in 2007. During the years 2008-2017, she worked in corporate engineering and management roles specializing in research and development of electronic engine control devices. She also served as a faculty member in Electrical Engineering, Arkansas State University (2017). She transitioned to biomedical applications related research in 2017 and has been pursuing research projects in the heart, liver, and brain. Her interests are control systems, physiological monitoring using electronic devices, modeling, and simulation of complex physical systems.

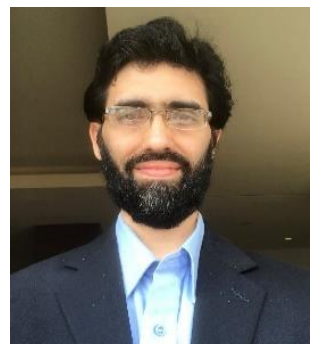

Viswanathan Rajagopalan completed BS degree at the Institute of Cardiovascular Diseases from Birla Institute of Technology \& Science (India) and Ph.D. in Physiology (Cardiovascular) from University of Nebraska Medical Center. He completed Postdoctoral training at Stanford University Medical Center (Cardiology) and was funded by a prestigious American Heart Association Grant. He is currently an Assistant Professor with New York Institute of Technology College of Osteopathic Medicine at A-State investigating novel aspects of cardiovascular diseases and is affiliated with AState's Center for No-Boundary Thinking.

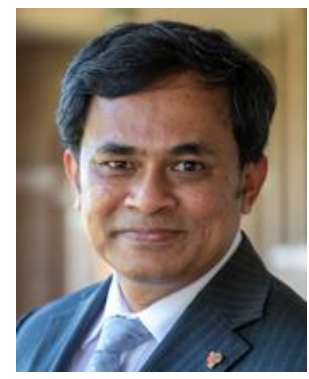

Bashir I. Morshed (M'12) received the B.Sc. degree in electrical and electronics engineering from Bangladesh University of Engineering and Technology in 2001, the M.Sc. degree in electrical and computer engineering from the University of Windsor in 2004, and the Ph.D. degree in electrical and computer engineering from Carleton University in 2010

Dr. Morshed is a recipient of the prestigious Canadian Commonwealth Scholarship in 2002. $\mathrm{He}$ is currently an Associate Professor with the Computer Science department of Texas Tech University since 2020. Previously, he was with the Electrical and Computer Engineering department of the University of Memphis since 2011. 